\title{
Ballistic Protons and Microwave-induced Water Solitons in Bioenergetic Transformations
}

\section{Reuven Tirosh}

The Biophysical Schottenstein Center, Physics Department, Bar Ilan University, Ramat-Gan 52900, Israel; E-mail: tiroshr1@ @ail.biu.ac.il; Tel: 972-9-767-9341

Received: 11 July 2006, in Revised Form: 21 September 2006 / Accepted: 22 September 2006 / Published: 26 September 2006

\begin{abstract}
Active streaming (AS) of liquid water is considered to generate and overcome pressure gradients, so as to drive cell motility and muscle contraction by hydraulic compression. This idea had led to reconstitution of cytoplasm streaming and muscle contraction by utilizing the actin-myosin ATPase system in conditions that exclude a continuous protein network. These reconstitution experiments had disproved a contractile protein mechanism and inspired a theoretical investigation of the AS hypothesis, as presented in this article. Here, a molecular quantitative model is constructed for a chemical reaction that might generate the elementary component of such AS within the pure water phase. Being guided by the laws of energy and momentum conservation and by the physical chemistry of water, a vectorial electro-mechano-chemical conversion is considered, as follows: A ballistic $\mathrm{H}^{+}$may be released from $\mathrm{H}_{2} \mathrm{O}-\mathrm{H}^{+}$at a velocity of $10 \mathrm{~km} / \mathrm{sec}$, carrying a kinetic energy of 0.5 proton*volt. By coherent exchange of microwave photons during $10^{-10}$ sec, the ballistic proton can induce cooperative precession of about 13300 electricallypolarized water molecule dimers, extending along $0.5 \mu \mathrm{m}$. The dynamic dimers rearrange along the proton path into a pile of non-radiating rings that compose a persistent rowing-like water soliton. During a life-time of $20 \mathrm{msec}$, this soliton can generate and overcome a maximal pressure head of $1 \mathrm{kgwt} / \mathrm{cm}^{2}$ at a streaming velocity of $25 \mu \mathrm{m} / \mathrm{sec}$ and intrinsic power density of $5 \mathrm{Watt} / \mathrm{cm}^{3}$. In this view, the actin-myosin ATPase is proposed to catalyze stereo-specific cleavage of $\mathrm{H}_{2} \mathrm{O}-\mathrm{H}^{+}$, so as to generate unidirectional fluxes of ballistic protons and water solitons along each actin filament. Critical requirements and evidential predictions precipitate consistent implications to the physical chemistry of water, enzymatic hydrolysis and synthesis of ATP, trans-membrane signaling, intracellular transport, cell motility, intercellular interaction, and associated electro-physiological function. Sarcomere contraction is described as hydraulic compression, driven by the suction power of centrallyoriented AS. This hydraulic mechanism anticipates structural, biochemical, mechanical and
\end{abstract}


energetic aspects of striated muscle contraction, leading to quantitative formulation of a hydrodynamic power-balance equation yielding a general force-velocity relation.

Keywords: ballistic protons, water solitons, hydraulic compression, physical chemistry of water, ATP hydrolysis, motor proteins, cytoplasm streaming, cell motility, muscle contraction, bioenergetics.

\section{Introduction: The Motor-Protein Paradigm and the Active-Streaming Hypothesis}

The phenomena of cytoplasm streaming and muscle contraction are generally related to enzymatic catalysis of ATP hydrolysis by the actin-myosin (A-M) system [1]. Therefore, by assuming a common driving mechanism, a simple question is raised: What comes first - streaming or contraction?

Up to 1954 , tension generation in striated muscle was related to contraction of the filamentous protein network within each sarcomere. However, during sarcomere shortening, the actin and myosin filaments where observed to slide past each other, while maintaining constant length. This seminal observation had actually revealed no protein contraction. Yet, the contractile-protein paradigm prevailed, being confined to a tensile force or a lever-like action by the A-M cross-bridges [2, 3]. The new hypothesis invited a terminological shift from "contractile proteins" to "motor proteins". This established view have raised minor interest in cytoplasm streaming, being considered as a passive flow caused by contraction of the A-M network.

Since 1972, the present author had advanced the investigation of a cause-effect reversal between streaming and contraction, by first raising the following argument: A mechanical protein action, unlike a fluid action, must rely on a continuous protein network. This argument was tested by reconstitution of protein transport, cytoplasm streaming and muscle contraction, utilizing the A-M ATPase system without forming a continuous protein network [4-13]. All these experiments had clearly revealed that the A-M system is uninvolved mechanically in tension generation. An early theoretical model for the alternative fluid mechanism was suggested $[14,15]$, as further developed in this article.

In a reciprocal relation to the hydrodynamic concept of passive streaming down pressure gradients, the new concept of active streaming (AS) is defined as being able to generate and overcome pressure gradients. The basic question is - what might drive AS within the pure water phase?

\section{A Proton-induced Water Soliton (pwason) (Fig.1, Suppl. Box 1, Suppl. Movies 1, 2)}

A vectorial electro-mechano-chemical transformation into AS in water is described by the classical laws of momentum and energy conservation, while quantitatively relying and even reflecting on the physical-chemical properties of liquid water. Consider the cleavage of a high-energy complex into two products of relatively small and large masses (m). By energy and momentum conservation, the two products gain an equal and opposite momentum (p), while the low-mass product carries most of the kinetic energy (Ek) released, namely:

$$
\mathrm{p}_{1}=-\mathrm{p}_{2} \rightarrow \mathrm{Ek}_{1} / \mathrm{Ek}_{2}=\left(\mathrm{p}_{1}^{2} /\left(2 * \mathrm{~m}_{1}\right)\right) /\left(\mathrm{p}_{2}^{2} /\left(2 * \mathrm{~m}_{2}\right)\right)=\mathrm{m}_{2} / \mathrm{m}_{1}
$$


The same laws, however, impose some restrictions that must be overcome for further efficient energy transfer from a ballistic product into massive streaming. First, molecular and electrical scattering must be avoided. Therefore, atoms and electrons are unsuitable products. A unique, electrically charged, product in water is a ballistic $\mathrm{H}^{+}$released from $\mathrm{H}_{2} \mathrm{O}-\mathrm{H}^{+}$. As shown below, the $\mathrm{H}^{+}$ kinetic energy is small enough to avoid also quantum-mechanical dissipation by electron excitation of surrounding molecules. Second, by the basic laws, a direct energy transfer into linear translation of massive streaming is not allowed. Therefore, electromagnetic transformation is considered to induce a persistent intermediate state of cooperative molecular circulation, which could drive AS by a rowinglike mechanism. Thus, by exchange of microwave photons, a ballistic proton is proposed to induce cooperative precession of many, electrically-polarized, water molecule dimers.

Dimer precession, rather than single molecular rotation, is considered for several reasons. First, by dimer precession, the proton kinetic energy is coherently shared by circulation of the whole mass of each water molecule. Second, the electrically-polarized dimers can reorganize to form non-radiating axial rings, so as to compose a persistent rowing soliton. Notice that the plane of circular precession is orthogonal to that of the rings, thus avoiding their disruption. Third, persistent cooperative precession can produce a peripheral rowing-like action, whereby the soliton might generate and overcome pressure gradients along the original proton path. Fourth, dimer precession must rely on intrinsic spin angular momentum. This spin is related by the virial theorem to a chain-like electrical interaction of the polarized dimers. Fifth, the frequency of dimer precession accounts for the unique mode of microwave absorption by liquid water. Thus AS might be driven by the inertia of molecular circulation rather than translation.

All these ideas precipitate major structural and energetic aspects of the pwason model. They are quantitatively evaluated in Supplementary Box 1, and further discussed below.

\subsection{The High-Energy Complex of $\mathrm{H}_{2} \mathrm{O}-\mathrm{H}^{+}$}

The free energy of $\mathrm{H}_{2} \mathrm{O}-\mathrm{H}^{+}$is obtained by the Maxwell-Boltzmann relation:

$$
\begin{gathered}
{\left[\mathrm{H}_{2} \mathrm{O}-\mathrm{H}^{+}\right] /\left[\mathrm{H}_{2} \mathrm{O}\right]=10^{-7} / 55.6=\mathrm{EXP}\left\{-\Delta \mathrm{E}_{1} / \mathrm{kT}\right\} \rightarrow} \\
\Delta \mathrm{E}_{1}=11.5 \mathrm{kcal} / \mathrm{mole}=0.5 \text { proton* volt }=8^{*} 10^{-13} \mathrm{erg}=20 \mathrm{kT} \quad \text { at } 20^{\circ} \mathrm{C}
\end{gathered}
$$

$\mathrm{H}_{2} \mathrm{O}-\mathrm{H}^{+}$is therefore a minor high-energy component of liquid water at thermal equilibrium. The $\mathrm{pH}-$ temperature dependence of liquid water is closely described by Eq.2. The de Broglie quantummechanical relation entails a classical view of a protonic molecular orbit enclosing the two lone-pair electrons of the oxygen atom in $\mathrm{H}_{2} \mathrm{O}-\mathrm{H}^{+}$(Figla). In this molecular view, a bound kinetic $\mathrm{H}^{+}$is held ready to be released upon cleavage of the parent $\mathrm{H}_{2} \mathrm{O}$ molecule. Supportively, dissociative photoionization of water reveals a minor fraction of ballistic protons, having a kinetic energy of up to 0.5 proton*volt [16].

\subsection{The electrically polarized dimers}

Water-molecule dimers are proposed to be the main, low-energy, component of liquid water. The molecular distances under interaction of two polarized dimers are presented in Fig.1a and in Supplementary Table1. This molecular structure is consistent with measurements of X-ray and neutron 
scattering [17], and with recent reports of X-ray absorption and emission spectroscopy, revealing proton delocalization [18], strong electron sharing [19] and double, rather than tetrahedral, hydrogen bonding between water molecules [20]. Theoretical simulation of liquid water has also uncovered a new role for 2-fold hydrogen bonds [21].

An extended electrically-polarized rigid dimer is required by the AS model. This molecular compound is proposed to rely on a central pair of double-hydrogen bonds, formed under covalent electron resonance of orthogonal $2 p$ molecular orbitals. The shorter end extensions of the electrically polarized dimer preserve the corresponding covalent configuration of a single water molecule dipole. The dimer dipole length $(0.42 \mathrm{~nm})$ is three times that of a free water molecule dipole $(0.14 \mathrm{~nm})$. Assuming the same electric charges of a water-molecule dipole $(1.8 \mathrm{D}$ along $0.14 \mathrm{~nm})$, the triple extension of the dimer configuration gives rise to an electric dipole of $5.4 \mathrm{D}$, which is effectively $2.7 \mathrm{D}$ per liquid water molecule, as measured. The dimer dipole extension strengthens short-distance electrical attraction between adjacent dimers through electrovalent double hydrogen bonds. This hybrid double hydrogen bond configuration allows both for internal spin and precession within each rigidly bound dimer, along with dynamic flexible orientation due to Coulomb attraction between dimers. All these features are required for cooperative precession of axial rings that may effectively compose the rowing soliton (Fig. 1b, Supplementary Movie 1). 


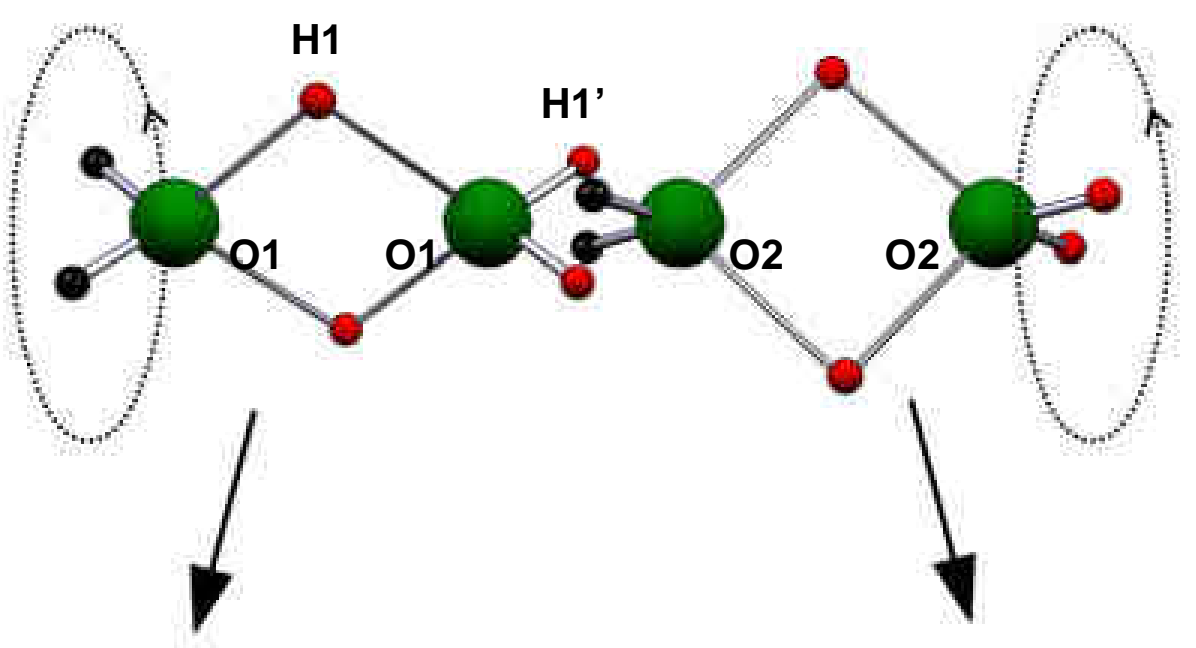

(a)
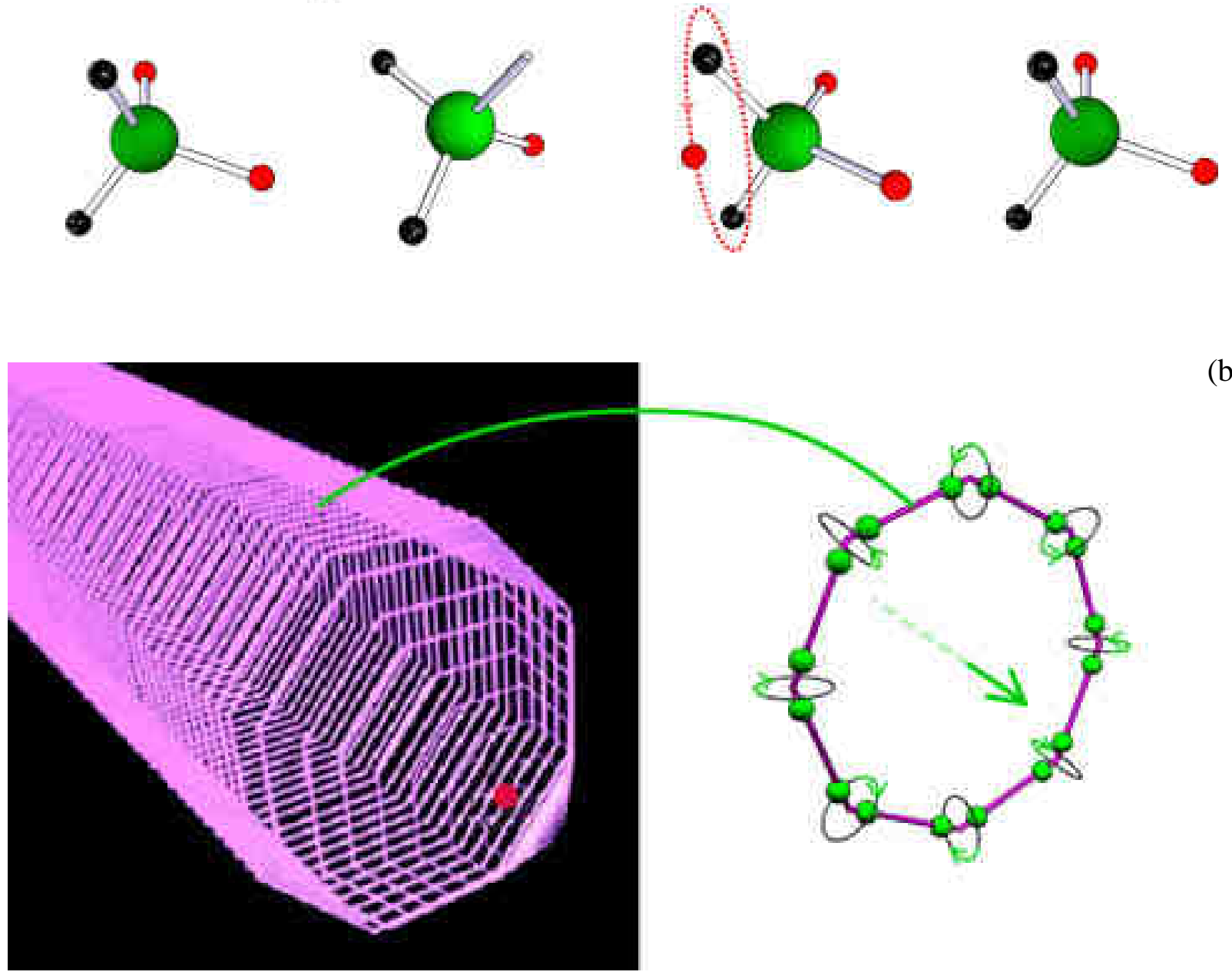

(b)

Figure 1. The water dimers and the pwason

(a) Internal spinning of two water molecule dimers, and their dissociation into water molecules and ions. Note the proton orbital in $\mathrm{H}_{2} \mathrm{O}-\mathrm{H}^{+}$.

$$
\mathrm{R}_{\mathrm{O} 1-\mathrm{O} 1}=\mathrm{R}_{\mathrm{H} 1-\mathrm{H} 1}=2.8^{*} 10^{-8} \mathrm{~cm}, \mathrm{R}_{\mathrm{H} 1{ }^{\prime}-\mathrm{H} 1^{\prime}}=\mathrm{R}_{\mathrm{O} 1-\mathrm{O} 2}=1.4 * 10^{-8} \mathrm{~cm}
$$

$$
\mathrm{R}_{\mathrm{O} 1-\mathrm{H} 1}=2 * \mathrm{R}_{\mathrm{O} 1-\mathrm{H} 1},=1.9 * 10^{-8} \mathrm{~cm}
$$

(b) Proton-driven precession of water-molecule dimers forming octal rings that compose a rowing soliton. 


\subsection{The dimer's spin and precession}

Dimer precession must rely on an intrinsic angular momentum, which is related to axial spinning of the four bound protons around the O-O axis (Fig.1a). A spin angular momentum of $25 \mathrm{~h}$ is obtained by the Virial Theorem, related to the electrical cohesive energy of double hydrogen bonding between water molecule dimers. Therefore, 25 states of dimer precession are anticipated, where in each state, the angular momentum of precession tends to counterbalance the projection of the spin angular momentum on the precession axis. Thus, at the highest energy state, this dimer precession has a total angular momentum of $1 \mathrm{~h}$ and kinetic energy of $6^{*} 10^{-17}$ ergs. These values correspond to the angular momentum and energy of microwave photons at a frequency of about $10^{10} \mathrm{sec}^{-1}$. In this state of dimer precession, each water molecule circulates at the maximal available radius, at an angular momentum of $\hbar / 2$, and at a velocity of $14 \mathrm{~m} / \mathrm{sec}$. (This dynamic state of dimer spin and precession is a vivid presentation of an intrinsic quantum-mechanical coupling between a boson and a couple of fermions, respectively.) The above values determine the $\mathrm{H}^{+}$flight duration of $10^{-10} \mathrm{sec}$, the path length of $0.5 \mu \mathrm{m}$, and the number of 13300 water molecule dimers that share the $\mathrm{H}^{+}$energy.

\subsection{The rowing soliton}

The soliton's active propulsion against tangential friction forces can generate a maximal pressurehead of $1 \mathrm{kgwt} / \mathrm{cm}^{2}$, corresponding to the kinetic energy density of water dimer precession. Compared to the gravitational hydrostatic pressure-gradient in water, the localized pressure-gradient within a single pwason is twenty million times greater $(10 \mathrm{~m} / 0.5 \mu \mathrm{m})$. The distant spreading of the dipolar pressure-gradient field of a single pwason is proposed to induce backward buoyant-like body forces and passive, fountain-like, streaming. This cooperative molecular mechanism of the soliton is incorporated into a reverse active version of the hydrodynamic laws of Archimedes, Bernoulli, Newton and Poiseuille, yielding for the pwason a streaming velocity of $25 \mu \mathrm{m} / \mathrm{sec}$ and a life-time of $20 \mathrm{msec}$.

\subsection{The pwason model}

The above arguments are integrated to construct the following model: Under spontaneous cleavage of the high-energy $\mathrm{H}_{2} \mathrm{O}-\mathrm{H}^{+}$complex, a ballistic $\mathrm{H}^{+}$is released at initial velocity of $10 \mathrm{~km} / \mathrm{sec}$, corresponding to a kinetic energy $\Delta \mathrm{E}_{1}=8^{*} 10^{-13} \mathrm{ergs}$. By coherent exchange of microwave photons of $6^{*} 10^{-17} \mathrm{ergs}$, the $\mathrm{H}^{+}$kinetic energy is transformed during $10^{-10} \mathrm{sec}$, along $0.5 \mu \mathrm{m}$, into cooperative precession of about 13300 electrically-polarized water molecule dimers. The dynamic polarized dimers rearrange into non-radiating octal rings, forming a persistent rowing soliton having a length of 500nm and a diameter of $1.4 \mathrm{~nm}$. During a lifetime of $20 \mathrm{msec}$, this rowing soliton develops a streaming velocity of $25 \mu \mathrm{m} / \mathrm{sec}$, while being able to generate and overcome a maximal pressure-head of $1 \mathrm{kgwt} / \mathrm{cm}^{2}$, with an intrinsic power density of $5 \mathrm{Watt} / \mathrm{cm}^{3}$. These quantitative features of the pwason model will be examined throughout this article. 


\section{Further Physical-Chemical Implications for Liquid Water}

3.1. In Scheme \#1, endothermic water dimer dissociation is related to the molecular ionization and evaporation energies $\left(\Delta \mathrm{E}_{2}, \Delta \mathrm{E}_{3}\right)$, which are revealed, respectively, by the heat released in the reverse processes of acid-base neutralization and water condensation.

$$
\begin{array}{ccc} 
& \text { Scheme 1 } \\
& & \\
\mathrm{H}_{2} \mathrm{O}-\mathrm{H}^{+}+\mathrm{OH}^{-} \stackrel{+2 * \Delta \mathrm{E}_{2}}{<==} & \mathrm{H}_{2} \mathrm{O}=\mathrm{H}_{2} \mathrm{O} \stackrel{+2 * \Delta \mathrm{E}_{3}}{=}=>\quad \mathrm{H}_{2} \mathrm{O}+\mathrm{H}_{2} \mathrm{O}
\end{array}
$$

$\Delta \mathrm{E}_{2}$ and $\Delta \mathrm{E}_{3}$ are approximately equal. Notably, the same molar value is also found for the free energy of $\mathrm{H}_{2} \mathrm{O}-\mathrm{H}^{+}$ions ( $\Delta \mathrm{E}_{1}$ in Eq.2, above), for ATP hydrolysis ( $\Delta \mathrm{E}_{4}$ in Eq.4, below), and for the heat + work per mole of ATP hydrolysis $\left(\Delta \mathrm{E}_{5}\right)$ as measured during closed cycles of muscle contraction [22]. Namely:

$$
\Delta \mathrm{E}_{1}=\Delta \mathrm{E}_{2}=\Delta \mathrm{E}_{3}=\Delta \mathrm{E}_{4}=\Delta \mathrm{E}_{5}=\Delta \mathrm{E}
$$

where $\Delta \mathrm{E}=8 * 10^{-13} \mathrm{ergs}=0.5$ proton $*$ volt $=48 \mathrm{kJoules} / \mathrm{mole}=11.5 \mathrm{kcal} / \mathrm{mole}$

3.2. The relatively high proton mobility in water is related to spontaneous release of ballistic protons from $\mathrm{H}_{2} \mathrm{O}-\mathrm{H}^{+}$. The life-time of a ballistic proton in water is related to the period of microwave photons in the range of $100 \mathrm{psec}$, as recently verified [23]. A longer range and life-time of proton mobility is anticipated within the ice phase, lacking microwave absorption by dimer precession.

3.3. A flow of water towards the cathode is anticipated under electric field application. This flow may be related to electrical orientation of spontaneous ballistic protons that induce AS.

3.4. The reversible function of a $\mathrm{pH}$ glass electrode in water is elucidated by a free passage of ballistic protons through a thin glass membrane, when protons are released from and recaptured on both sides as $\mathrm{H}_{2} \mathrm{O}-\mathrm{H}^{+}$ions, yielding the $\mathrm{pH}$-indicative electric potential at thermal equilibrium. A similar electrochemical performance is expected across biological membranes, where passage of ballistic protons might induce transient gating of fluid, molecular, and ionic currents [24].

3.5. The first water anomaly, i.e. volume contraction upon ice melting, may be due to a transformation from tetrahedral hydrogen bonding to the more compact packing of the electrically polarized dimers. This phase transition elucidates the anomalous phenomenon of high and low density states of water and of ice, at lower temperatures and under higher pressures [25, 26].

3.6. The 25 energy states of dimer precession predict a testable thermal ladder for water between 0 and $100{ }^{\circ} \mathrm{C}$. If evenly spaced every $4{ }^{\circ} \mathrm{C}$, this ladder might explain the second anomaly of water. This thermal ladder might also explain the unexpected thermal anomalies seen in the temperature responses of living systems at about every $16{ }^{\circ} \mathrm{C}$ increments [27].

3.7. There are two easy ways to grasp the soliton's pressure-head of $\Delta \mathrm{P}=1 \mathrm{kgwt} / \mathrm{cm}^{2}=1 \mathrm{kgwt} * \mathrm{~cm} / \mathrm{cm}^{3}$, by stemming from the water molecule precession velocity, $\mathrm{V}=14 \mathrm{~m} / \mathrm{sec}$. First, this $\Delta \mathrm{P}$ value is derived 
as the kinetic energy density, namely $\Delta \mathrm{P}=1 / 2 \rho^{*} \mathrm{~V}^{2}$, where $\rho=1 \mathrm{gr} / \mathrm{cm}^{3}$. Second, the same pressurehead is produced under gravitation by a $10 \mathrm{~m}$ water column, where such a column can be created by upward water flow with an initial velocity of about $14 \mathrm{~m} / \mathrm{sec}$.

3.8. By the same reasoning, if surface winds drive long-distance sea waves due to cooperative energy absorption by water dimer precession, then such waves may reach maximal amplitude of $10 \mathrm{~m}$, or peakto-peak height of $20 \mathrm{~m}$ [28]. (Standing waves in closed straits might reach twice this amplitude). Cooperative molecular inertia of water dimer precession at $\mathrm{Vw}=14 \mathrm{~m} / \mathrm{sec}=50 \mathrm{~km} / \mathrm{hour}$, may also account for the velocity of large-scale ocean waves, as well as for their vertical circulation profile. The same mechanism is related to generation and propagation of a water solitary wave following abrupt stopping of a ship in a narrow canal [29]. The same mechanism is proposed for microscopic Bekesy waves, propagating at $14 \mathrm{~m} / \mathrm{sec}$ along the cochlear canal of the ear [30]. Similarly, microscopic turbulence can cause abrupt changes in micro-particle velocities up to $14 \mathrm{~m} / \mathrm{sec}$ [31]. Thus, the liquid water phase mediates diverse macroscopic and microscopic effects that are quantitatively related to the quantum of water dimer precession.

3.9. In this view, it is tempting to speculate that even the atmospheric pressure of $1 \mathrm{kgwt} / \mathrm{cm}^{2}$ at sea level may manifest a balanced steady state with surface generation of pwasons.

3.10. Furthermore, the spontaneous surface ejection of kinetic protons might leave the oceans at a negative electric potential. Trapped in the atmospheric vapor, the protons can be involved in the cohesive dispersion of clouds. Various lightening effects might therefore be due to massive $\mathrm{H}^{+}$ currents.

3.11. Similarly proton-driven micro-atmospheric lightening effects are presumably manifested in single-bubble sono-luminescence in water [32]. Note that the ambient $20 \mathrm{kT}$ ballistic proton is equivalent to a thermal plasma nucleon at $20^{*} 300^{\circ} \mathrm{K}=6000^{\circ} \mathrm{K}$.

\section{Biochemical implications for the Actin-Myosin (A-M) system}

Unidirectional vectorial fluxes of protons and solitons may be generated along a single actin filament by stereospecific cleavage of $\mathrm{H}_{2} \mathrm{O}-\mathrm{H}^{+}$. Enzymatic catalysis of this reaction is proposed for ATP hydrolysis by myosin heads, while being attached to the actin filament. The reaction may take place at the P-loop of the apical side, on the molecular clefts of myosin heads attached to actin [33] (Fig.2a). The mechano-chemical proton-burst reaction occurs concomitantly with hydration of the terminal P-O-P bond, as given by:

$$
\mathrm{ADP}-\mathrm{O}-\mathrm{P}^{-4}+\mathrm{H}_{2} \mathrm{O}-\mathrm{H}^{+} \rightarrow \mathrm{ADP}-\mathrm{O}-\mathrm{H}--\mathrm{O} H-\mathrm{P}+\mathrm{H}^{+}+\Delta \mathrm{E}_{4}
$$

According to Eq.1, the kinetic proton carries the free energy $\Delta \mathrm{E}_{4}$, which is equal to $\Delta \mathrm{E}$ in Eq.3. The ATP anion is proposed to interact with $\mathrm{H}_{2} \mathrm{O}-\mathrm{H}^{+}$, rather than $\mathrm{H}_{2} \mathrm{O}$ as generally accepted. Indeed, the rate of spontaneous ATP hydrolysis increases in acidic solutions. 
The enzymatic cycle for the A-M system of striated muscle is described in Scheme\#2. It is consistent with structural, kinetic, and isotopic measurements [34-36]. The enzymatic cycle is described in three steps between three states:

\section{Scheme 2}

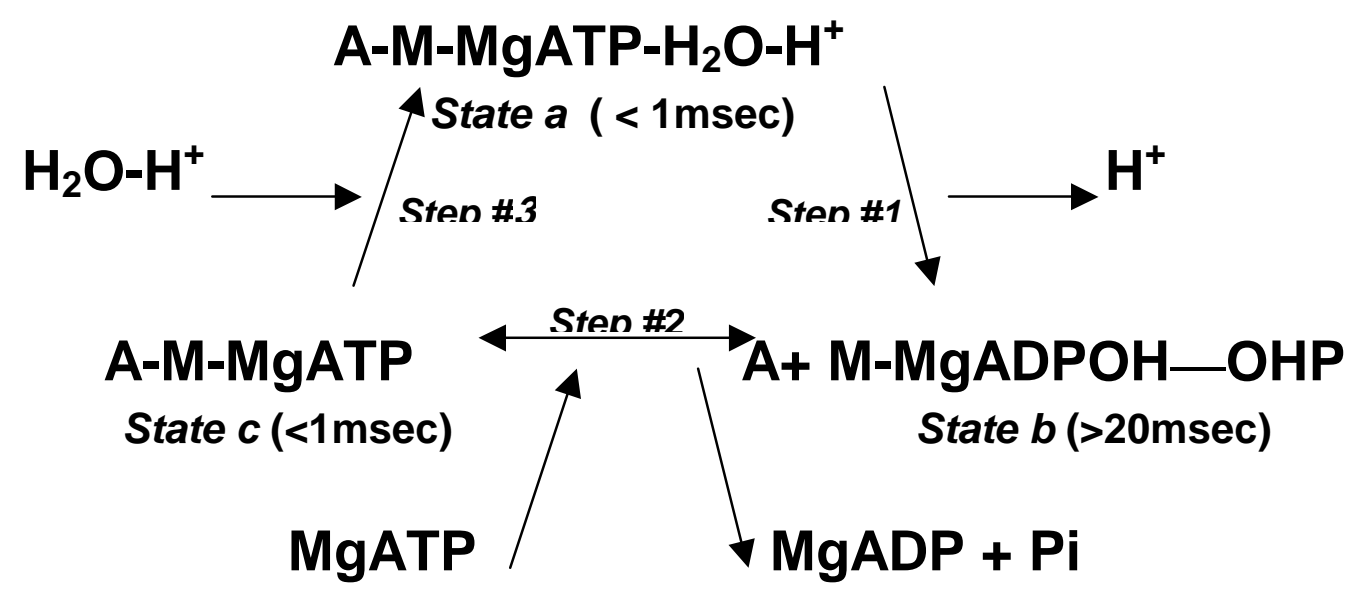

State $a$ is a short-lived high-energy stereo-specific rigid complex. The stereo-specific configuration might rely on the quaternary ionic complex of $\mathrm{Mg}^{+2}$ with $\mathrm{ATP}^{-4}$ that is proposed to maintain a ring-like rigid framework of the adenine-ribose-triphosphate complex. This complex is attached to the P-loop of the catalytic site, which is exposed to the water phase, opposite to the A-M binding site. At Step \#1, the $\mathrm{H}^{+}$ejection occurs concomitantly with an equal recoil momentum and with an abrupt increase of negative charge at the myosin head. Both effects enhance the detachment of a stable product-loaded myosin head. In State $b$, the pwason remains active during $20 \mathrm{msec}$, while preventing effective reattachment of the myosin head to actin. This dominant state of protein dissociation during the enzymatic cycle ensures minimal resistance to filaments sliding. In Step \#2, an effective A-M binding enhances product-substrate exchange under thermal equilibrium. Thus, the release of ADP and the inorganic phosphate $\mathrm{Pi}$ is delayed after completion of the hydraulic power stroke [37]. (Step \#2 is regulated by the troponin-tropomyosin system at micromolar concentrations of calcium ions.) The duration of State c, at physiological millimolar concentrations of MgATP, is relatively short. Indeed, the duration of the A-M complex is estimated to hold less than $5 \%$ of the enzymatic cycle period.

This cyclic interaction profile is considered for other types of myosin that determine the pwasons direction along the actin filament [38-40]. A similar mechanism based on proton-induced water solitons is suggested for ATP hydrolysis by dynein or kinesin along microtubules, or by G-actin polymerization [41], as well as for other substrates of various hydrolytic enzymes. Thus, a hydraulic drive due to AS is proposed to translocate the ATPase machinery of DNA replication, RNA transcription, or peptide translation. These processes can be effectively associated with active flow of substrates and products across the catalytic regions.

The microwaves proton-soliton coupling is irreversible. For a reversible reaction of ATP hydrolysis, the ballistic protons must be separated from the water phase, as discussed below. 


\section{Biochemical implications for the reversible function of membrane-bound ATPases}

The proton-burst equation (Eq. 4) suggests a reversible pathway for ATP synthesis. In the direction of synthesis, a ballistic $\mathrm{H}^{+}$could strike a catalytic site in-between the juxtaposed anions of ADP and Pi. This $\mathrm{H}^{+}$impact might extract the high-energy complex of $\mathrm{H}_{2} \mathrm{O}-\mathrm{H}^{+}$, while leaving attached the anhydride ADP-O-P complex. However, this dehydration pathway requires a hydrophobic compartment in order to uncouple the proton movement from AS induction. In the absence of solitons interference at the hydrophobic catalytic region, a much faster reversible catalysis is anticipated, compared to the soluble A-M system. Such a reversible ballistic proton mechanism may be catalyzed by the membrane-bound $\mathrm{F}_{\mathrm{O}}-\mathrm{F}_{1}$ ATP-synthase of bacteria, mitochondria and chloroplasts, as discussed by the following arguments.

5.1. The proposed mechanism involves two main aspects. First, the trans-membrane concentration difference of $\mathrm{H}_{2} \mathrm{O}-\mathrm{H}^{+}$determines the direction and rate of the ballistic proton flux and the corresponding amount of ATP synthesis or hydrolysis. Second, in the direction of ATP synthesis, a threshold electric potential difference is obligatory for compensation of a certain dissipation of the proton's kinetic energy. (e.g. with a total energy of 0.5 proton*volt, 0.1 volt compensates for $20 \%$ loss). Thus, the ballistic proton mechanism elucidates the thermodynamic concept of a "proton-motiveforce" in the chemiosmotic hypothesis $[42,43]$. It accounts quantitatively for the elementary energetic event; it bypasses the problem of trans-membrane proton transportation; and it differentiates between independent roles of chemical potential and electrical potential of $\mathrm{H}_{2} \mathrm{O}-\mathrm{H}^{+}$across the membrane [44].

5.2. Furthermore, rotational catalysis was proposed for the $F_{O}-\gamma \varepsilon-(\alpha \beta)_{3}-F_{1}$ complex [45]. This rotation might be electrically driven by the reversible ballistic proton mechanism, as follows. In ATP synthesis, each ADP-Pi loaded $\alpha \beta$-site of the water exposed $F_{1}$ head is bound in turn to the hydrophobic, topically bent, $\gamma \varepsilon$ axis. This internal axis is inserted into the $\mathrm{F}_{\mathrm{O}}$ membrane component so as to form an effective channel for ballistic protons. The $\alpha \beta$ catalytic unit is comparable to a myosin head, while the biochemical role of the $\gamma \varepsilon$ axis is quite similar to that of the actin filament in the enzymatic cycle (Scheme \#2). Thus, in the direction of synthesis, the impact of a trans-membrane ballistic $\mathrm{H}^{+}$within the hydrophobic catalytic region is proposed to drive three concomitant effects: First, dehydration of the terminal phosphate bond results in ATP synthesis. Second, molecular recoil upon the $\mathrm{H}^{+}$impact dissociates the $\gamma \varepsilon-\alpha \beta$ complex. Third, the abrupt increase of electric charge at the hydrophobic site drives fast relative rotation at $120^{\circ}$ towards hydrophobic $\gamma \varepsilon$ interaction with the next, ADP-Pi loaded, $\alpha \beta$-site. Simultaneous exchange of products and substrates, carried out at the other two, water exposed, $\alpha \beta$ sites, might electrically dictate ongoing rotation in the appropriate direction.

Inversely, opposite rotation might be similarly driven under ATP hydrolysis, while generating transmembrane efflux of ballistic protons, which are trapped as $\mathrm{H}_{2} \mathrm{O}-\mathrm{H}^{+}$ions. Thus, the reversible ballistic proton mechanism can consume or generate trans-membrane electro-chemical potential of $\mathrm{H}_{2} \mathrm{O}-\mathrm{H}^{+}$ ions. In this view, the proton-induced electric drive of rotation is considered to share a minor part in energy consumption, compared to the lion share of P-O-P dehydration in ATP synthesis, or of building up the trans membrane electrochemical gradient of $\mathrm{H}_{2} \mathrm{O}-\mathrm{H}^{+}$under ATP hydrolysis. 
5.3. Stepwise rotation was first demonstrated by attaching a relatively long actin filament to an exposed $\gamma$ axis of a fixed $F_{1}$ head [46]. For each hydrolytic event, the long flexible filament exhibited a fast $1 \mathrm{msec}$ step of unbent rotation through $120^{\circ}$, followed by a pause during about $20 \mathrm{msec}$. Such a fast rotation step may be easily driven by a single pwason, acting along $500 \mathrm{~nm}$ during a lifetime of $20 \mathrm{msec}$. Therefore, the observation of fast $120^{\circ}$ steps of rotation, while preserving radial extension of the long flexible filament, should be considered as another compelling evidence for the AS hypothesis.

Molecular demonstration of ATP synthesis was carried under artificially-forced opposite rotation of the $\gamma \varepsilon$ axis [47]. This rotation is considered to re-occupy the hydrophobic compartment with a MgADP-Pi loaded $\alpha \beta$-site. Thereby, the hydrophobic catalytic site is being prepared toward spontaneous impact of a ballistic proton for ATP synthesis.

5.4. The reversible ballistic proton mechanism is directly acting at the intact catalytic site. In this view, there is no need for intermediate power transmission between two motor proteins, as currently assumed for the $\mathrm{F}_{\mathrm{O}}$ and $\mathrm{F}_{1}$ components. Like the $\mathrm{F}_{\mathrm{O}}-\mathrm{F}_{1}$ system, the reversible rotation of bacterial flagella under trans-membrane $\mathrm{pH}$ difference [48] could be driven by molecular electrostatic forces, as induced by the ballistic proton mechanism. Thus, intermittent switching between opposite rotations could be controlled by switching between opposite $\mathrm{pH}$ gradients.

5.6. The biochemical similarity between the A-M and the $\mathrm{F}_{\mathrm{O}}-\mathrm{F}_{1}$ systems, as well as other soluble and membrane-bound ATPases, is revealed in their utilization of the quaternary MgATP complex within the P-loop catalytic site [49]. This evolutionary conserved framework is probably required for a stereospecific interaction that ensures an appropriate channeling of the ballistic protons. Thus, in reconstitution experiments in the absence of $\mathrm{Mg}$ ions, ATP hydrolysis by A-M or $\mathrm{F}_{\mathrm{O}}-\mathrm{F}_{1}$ is carried out as intensively as by $\mathrm{M}$ or $\mathrm{F}_{1}$ alone, but producing no myofibril contraction or outward trans-membrane flux of protons, respectively. In such reconstitution experiments, the $\gamma$-axis rotation on the $F_{1}$ head is still present under hydrolysis of CaATP [50]. This demonstration is consistent with the ballistic proton mechanism, where the electrostatic rotational drive is not the main energy consumer.

5.7. Trans-membrane fluxes of ballistic protons through specific channels could induce a flow of water, together with specific, loosely bound, molecules and ions, moving up or down gradients of hydrostatic pressure, chemical concentration, and electric potential. Like the $\mathrm{F}_{\mathrm{O}}-\mathrm{F}_{1}$ system, these proton-driven pumps may be coupled in closed vesicles to membrane-embedded redox and photonic reactions that produce, or consume, trans-membrane changes in $\mathrm{pH}$ and electric potential. This mechanism is related to the photosynthetic pathway of bacterio-rhodopsin, to visual signaling by rhodopsin, and to photosynthetic water oxidation in thylakoids [51]. Various signaling pathways are associated with the hydration or dehydration of phosphate bonds that may be related to a release or absorption of ballistic $\mathrm{H}^{+}$from or onto $\mathrm{H}_{2} \mathrm{O}-\mathrm{H}^{+}$. Through changes in $\mathrm{pH}$ and/or electric potential, transmembrane signaling by ballistic protons might trigger various physiological effects, such as calcium ion mobilization from internal or external sources. 


\section{Molecular and cellular implications for the A-M system}

\subsection{Molecular motility assays and intracellular transport}

The development of AS along a free actin filament is predicted to drag the filament along with attached myosin heads. Such a random motion of individual actin filament "rockets" in dilute solution was verified by Doppler broadening of laser-light scattering [13]. A similar drag of a free actin filament in one or the other direction is demonstrated also when the myosin molecules are anchored. By immobilizing the actin filament, the myosin heads might be propelled along with AS. However, a microscopic cargo attached to a myosin head might rather be pushed down the pressure gradient $(\operatorname{grad} \mathrm{P})$ due to AS. (According to the relation $\mathrm{F}=\mathrm{V}^{*} \operatorname{grad} \mathrm{P}$, a buoyancy-like hydraulic body force $-\mathrm{F}$ is proportional to the body volume - V.) In a single hydrolytic event, this hydraulic force could pull the myosin head along the actin filament in a stepwise sliding movement due to occasional A-M interactions that remain catalytically ineffective, as observed [52]. The integral duration and extent of this stepwise drag due to a single hydrolytic event further verify the theoretical values for a single pwason, namely - its 20msec life-time and its possible extension up to 500nm [53]. In the case of double-head myosin, a hand-over-hand drag along actin is anticipated. Such "walking" movement of the double-head Myosin VI reveals a single head stepping of $60 \mathrm{~nm}$, which is much larger than its 10 $\mathrm{nm}$ lever arm. This demonstrates sufficient power of AS to stretch the stalk's dimeric twist [54, 55].

Further evidence for the pwason model is gained by several observations that confirm the prediction of unidirectional drag movement by AS and dynamic electrical polarization of actin filaments due to the ballistic proton flux (Fig.2a), as discussed below:

6.1.1. A free electrically-polarized actin filament, moving under AS, might tend to reorient in the direction of a relatively small electric field, as observed [56]. Indeed, due to the electric force acting on their net negative charge, the filaments moving towards the anode go faster than without an external field, while those moving towards the cathode go slower, as expected.

6.1.2. Attractive hydrodynamic and electrical polarization forces among soluble actin filaments, are expected to orient the filaments so as to drive massive cytoplasm streaming. An imitation of such massive streaming, as observed in dumbbell-shaped micro-plasmodia of Physarum, was reconstituted in stretched micro-capillaries [4].

6.1.3. Lateral hydraulic compression, due to Bernoulli's effect of AS, can form and maintain a narrow channel in the dumbbell-shaped micro-plasmodia of Physarum. Notably, the velocity of AS across the elongated channel might reach up to $1 \mathrm{~mm} / \mathrm{sec}$, which is much greater than the pwason's theoretical value of $25 \mu \mathrm{m} / \mathrm{sec}$. This vital increase in streaming velocity in proportion to the length of the streaming pathway is simply explained as follows: serial pwasons generate the same pressure head $(\Delta \mathrm{P})$ along a greater distance $(\mathrm{L})$, thus reducing the pressure gradients $(\Delta \mathrm{P} / \mathrm{L})$ that oppose the AS. Similarly, a relatively high drag velocity is predicted for elongated actin filaments, as observed in motility assays [57].

6.1.4. Shuttle cytoplasm streaming along a narrow channel of Physarum, with a period of about two minutes, was recorded by simultaneous kinetic measurement of two oscillating variables: the opposing 
pressure head, which is required to stop it, and the autonomic buildup of a phase-lagged electric potential difference, referred to as the "electro-dynamo-plasmo-gram" [58]. Each streaming reversal occurred at the peak of the electric field, and it was associated with a concomitant record of a nervelike action potential. This complex profile is simply explained by unidirectional association of protondriven AS along oriented actin filaments, and the autonomic development of electric field, until it induces a cooperative flipping of the electrically polarized actin filaments.

6.1.5. A similar cytoplasmic mechanism was therefore considered for bulk generation and propagation of a nerve action potential and for the hydraulic release of neurotransmitters from synaptic vesicles [59]. Thus, in a neuron at rest, a threshold flux of protons and solitons towards the cell body may be generated along oriented actin filaments in the dendrites and the axon. A negative electric potential and a lower hydrostatic pressure are thereby maintained at the nerve terminals. Stimulating signals at the dendrites will enhance proton fluxes that will integrate to increase and even positively reverse the electric potential at the cell body. A local strong electric field is thereby created at the axon entrance, where it might locally flip over the electrically polarized actin filaments. Concomitantly, a localized increase in electric potential and in hydrostatic pressure might develop by opposing fluxes of protons and solitons. The localized increase in electric potential is further regenerated by gating the sodium ion influx, thus safely propagating the action potential down the axon under gradual flipping of the actin filaments. Upon reaching the nerve terminal, a momentary increase of hydrostatic pressure might induce a hydraulic release of neurotransmitters from the terminal vesicles. Excess reuptake is driven by sub-pressure recovery due to reorientation of the actin filaments. According to this mechanism, the action potential is predicted to involve bulge propagation and longitudinal compressive tension due to AS (like in a virtual sarcomere), as was indeed observed [60]. Furthermore, associated impulses of birefringence reduction, $\mathrm{pH}$ decrease, increase in $\mathrm{Ca}^{2+}$ ion concentration, and a rise of heat production, are anticipated to propagate along with the action potential, as observed [61]. In sensory organelles, similar mechanisms might induce electro-mechanical modulation of bulk proton currents and AS along oriented actin filaments [62].

6.1.6. Fish electric organ is composed of $100 \mu \mathrm{m}$ units, containing unidirectional actin filaments [63]. Coherent stimulation of a bulk proton flux, that can develop 0.5 volt per each serial unit, might generate electric shock impulses of up to $5000 \mathrm{volt} / \mathrm{m}$.

6.1.7. Similarly, the localized stimulation of centrally innervated myofibrils might autonomously propagate down the non-innervated regions through differential proton-induced depolarization along the sarcomeres.

\subsection{Cell motility and intercellular interaction}

Pwason fluxes integrate along oriented actin filaments into bulk AS, pressure gradients, and electrical polarization, all in the same direction, as verified also at the cellular level in the following examples. 
6.2.1. Amoeboid-like movement is electrically polarized in the direction of streaming [64]. A higher pressure is developed at the anterior area and a sub-pressure at the posterior region [65] (Fig.2b). The forward pressure-gradient can be responsible for three observable effects: frontal protrusion, peripheral fountain-like backward streaming, and either flattening or permanent endocytosis at the posterior region [66]. In case of ground adhesion, the suction power of forward AS can "weigh anchor" by hydraulic thinning and tearing of rear cytoplasmic fibers.

6.2.2. Adjacent electrically-polarized motile cells are predicted to move in a head-to-tail orientation. Upon cells contact, unidirectional AS can generate hydraulic conjugation through dynamic intercellular invaginations (Fig.2c). A forward proton flux through cognitive receptor channels can preferentially stimulate the leading cell, where intensive AS is exhibited. In cytotoxic T-lymphocyte interaction, stimulation of such AS in the target cell is observed as aggressive terminal explosion, termed Zeiosis [67].

6.2.3. Linear and branched polymerization of actin filaments attached to the plasma membrane, or to internal components, is associated with ATP hydrolysis that can locally generate AS and pressure gradients [68]. Similarly, antigenic receptor-mediated cell stimulation is related to internal clustering of membrane-anchored actin filaments, which induce localized orthogonal pwasons. Hydraulic endocytosis can down-regulate this cellular stimulation, which is subsequently communicated by AS to internal organelles in various intracellular pathways. Antibody + complement fixation of the stimulatory antigenic complexes can inhibit this hydraulic endocytosis, thus leading to lethal metabolic exhaustion [69]. Such hydraulic action is indicated in reconstitution experiments of nano-tube extraction from membrane vesicles by kinesin ATPase activity along microtubules [70].

6.2.4. Similarly, in erythrocytes, trans-membrane ventilation of $\mathrm{CO}_{2}$ and $\mathrm{O}_{2}$ may be enhanced by subpressure fluctuations, generated by inwardly oriented pwasons due to ATPase activity of branched actin polymerization on membrane-attached filaments [71]. According to the pwason model, the rate and amplitude of these active membrane fluctuations are anticipated also for other membrane-attached hydrophilic ATPases [72]. Such hydraulic compression of the cell membrane might explain the peculiar observation of a higher internal hydrostatic pressure in an intact cellular state of partial inflation. Intracellular vesicles, bacteria or designed objects can utilize this hydraulic machinery for the actin-based propulsion inside the cell or in a cell-like medium [73].

6.2.5. The A-M gel state of amoeboid cells at rest is observed to break down under the power of AS. A similar gel-sol transition is observed in-vitro in A-M preparation from striated muscle at $\mathrm{mM}$ concentration of MgATP. At diminishing levels of MgATP, A-M clusters are formed, demonstrating vigorous dehydration, termed super-precipitation [1]. This process of water squeezing is related to a localized hydraulic compression of each protein cluster under the suction power of AS.

6.2.6. Centrosomes' and chromosomes' separation during mitosis is related to viscous drag by opposite outwardly oriented AS due to cooperative action of various ATPase systems [74]. Consequently, central sub-pressure might cause cellular cleavage by equatorial hydraulic compression. 
6.2.7. Motility assays of free actin filaments verify that AS in striated muscle is generated toward the sarcomere's center. The suction power of centrally-oriented AS in a smooth muscle cell, or in each sarcomere of striated muscle, might generate sub-pressure at the opposite end regions of each unit, leading to its hydraulic compression, as elaborated below.
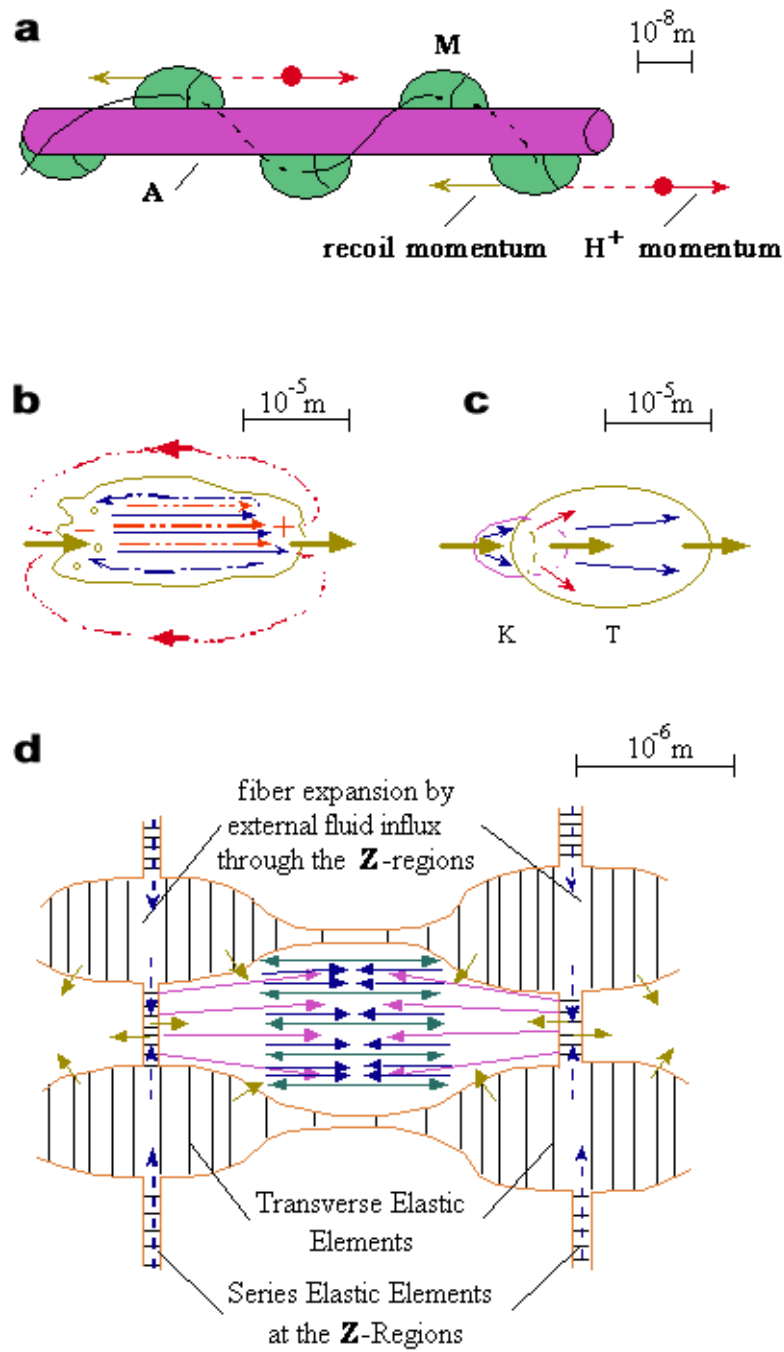

Figure 2. Ballistic protons and water solitons in biology

(a) Vectorial flux of protons from myosin (M) heads along actin (A) filament.

(b) Unidirectional electrical polarization and active streaming against pressure gradients in amoeboid movement.

(c) Hydraulic conjugation and trans-membrane protonic stimulation in killer-target (K-T) interaction.

(d) Hydraulic compression and lateral expansion of a muscle sarcomere due to centrally oriented active streaming.

\subsection{Hydraulic compression of the sarcomere}

Essential structural features of striated muscle fibers and significant physiological functions are further anticipated by the proposed mechanism of hydraulic compression due to active streaming: 
6.3.1. The primordial pwason dimensions - $1.4 \mathrm{~nm}$ diameter and $0.5 \mu \mathrm{m}$ length - explain the spatial evolution of optimal packing of sliding filaments in the sarcomere. The persistent hydrodynamic action throughout the pwasons' lifetime ensures minimal friction and efficient mechano-chemical performance by preventing cross-bridges interaction during most of the enzymatic cycle, while preserving the individual straight extension of the sliding filaments. This simply means that only a very small fraction of myosin heads are attached to actin filaments at any single time during contraction. This dynamic state under detachment of the A-M filaments is recognized in X-ray interference reports, where it is considered to be highly problematic in terms of the cross-bridge mechanism [75].

6.3.2. The hydraulic compression of each sarcomere requires an efficient peripheral buffering of hydrostatic pressure inside the Z-regions. This pressure buffering is related to intensive plasma membrane invaginations, which penetrate at the level of the Z-regions and extend around the I-regions of each sarcomere [76]. The cellular development and maintenance of this micro-fluidic network might itself be due to hydraulic AS processing. The serial dense elastic elements at the Z-regions hold the hydraulic compressive tension. Similarly, in order to resist lateral hydraulic compression, transverse elastic elements must be formed alongside between adjacent sarcomeres. These transverse elements also preserve the orthogonal invagination pattern which is essential for the hydraulic compression mechanism.

6.3.3. According to the hydraulic model, the isometric tension is held by a series of elastic elements at the Z-regions. Therefore, no forces, comparable to the isometric tension, are expected to stretch the actin and myosin filaments. This prediction is verified by X-ray interference measurements during fast force transients [77]. The absence of filament stretching under tension is also revealed in the sarcomeres' wave-like pattern that is developed under isometric contraction. (see Ref.3, p.332, Fig.20,I). This non-stretched profile under tension is a paradox in conventional terms, but it is an important prediction directly derived from the hydraulic mechanism. Its implications will be further discussed below.

6.3.4. Hydraulic suction of external water influx through the transverse network of plasma membrane invaginations is expected during isometric contraction. This influx allows for the ongoing overall expansion under lateral compression of the I-regions, while stretching the transverse elastic elements. Concomitantly, the longitudinal pumping action by AS causes a central inflation of each sarcomere (Fig.2d). This swelling mechanism explains the observed wave-like (barrel-like) pattern mentioned above, whereby tubular sarcomeres become constricted at the I-regions. This active swelling process is proposed to have four most significant physiological functions:

6.3.5. First, during isometric tetanus, an early development of an approximately maximal pressure-head tension of $\mathrm{Po}=1 \mathrm{kgwt} / \mathrm{cm}^{2}$, is followed by an ongoing slower expansion of the cross-section area, from Ao to Ae. This expansion process entails a proportional increase of the hydraulic force up to $\mathrm{Fe}=$ Po.Ae. Usually, the effective tension, Teff $=\mathrm{Fe} / \mathrm{Ao}=\mathrm{Po} \cdot \mathrm{Ae} / \mathrm{Ao}$, is reported. Thus, the Teff value may reach more than twice the actual Po tension $[15,78]$. A similar increase in isometric force is obtained under osmotic expansion [79]. 
6.3.6. Second, the pressure-head development and relaxation precede the slower effects of initial fiber swelling and terminal elastic deflation, respectively. Thereby, three effects are anticipated for isometric force development, known as the creep phase, post-tetanic potentiation, and stretch potentiation. When these effects are taken into account, a nearly flat force-length relation is obtained, which is quite independent of the degree of cross-bridges' overlap [15, 80]. This creep-phase effect is another fundamental problem for the established model,.

6.3.7. Third, the swelling process can provide hydraulic protection against series stretching of weaker sarcomeres, especially at a decreasing cross-bridges overlap. Evidently, and quite luckily, a homogenous steady state is maintained [81]. (Notice that by a cross-bridge mechanism, a "tearing catastrophe" is rather anticipated.) This protective mechanism is demonstrated by a whole muscle response during isotonic contraction, when the load is suddenly increased somewhat above the isometric tension; then, an initial "give" effect of rapid elongation slows down, stops, and even overcomes the initial overload [82]. Such a protective hydraulic response is obviously important also in cardiac and smooth muscle contraction.

6.3.8. Fourth, repetitive contraction is associated with pumping of external fluid fluxes around each sarcomere. These external fluid pulses, together with internal circulation of AS, provide for an effective metabolic exchange and heat dissipation, over and above the slow rates of diffusion and convection. Evidently, a relatively quick fatigue is felt under isometric tetanus.

6.3.9. Similarly, the internal circulation of AS in each sarcomere might enhance the regulatory function of $\mathrm{Ca}^{2+}$ ions in inverse relation to the muscle power. Thus, a suitable response rate is autonomously secured upon contraction and relaxation.

6.3.10. By initial coherent stimulation of AS, a transient lateral compression, due to the Bernoulli Effect, entails the well-known early events of latency elongation and relaxation of myofibrils. Thus, by Scheme\#2, under photo-release of caged ATP at sub-threshold levels of $\mathrm{Ca}^{2+}$ ions, a single pulse of coherent pwasons can generate an early isometric relaxation, followed by a $20 \mathrm{msec}$ phase of hydraulic compression throughout the ongoing state of protein dissociation, as observed [83].

6.3.11. Coherent interaction of cross-bridges might occasionally interfere with filaments sliding, as revealed in histograms of stepwise shortening and elongation [84]. In skeletal muscle, this resistive interaction is smoothed out by a differential Vernier scaling of monomers with different lengths between the sliding filaments.

6.3.12. In contrast, monomers of equal lengths are found in the sliding filaments of asynchronous insect flight muscles, which exhibit a slow rate of $\mathrm{Ca}^{2+}$ reuptake by the sarcoplasm reticulum. Therefore, a relatively low frequency of stimulation can maintain persistent $\mathrm{Ca}^{2+}$ activation, where simultaneous cross-bridges attachment might catalyze bulk coherent pulses of pwasons. Such repetitive coherent pulses of AS might generate fast and efficient cycles of hydraulic compression and relaxation. This pulsating contraction has a relatively large amplitude of $70 \mathrm{~nm}$ per half sarcomere, with no interference of cross-bridges' interaction all along the period of each cycle [85]. 
6.3.13. The established sliding filament model relies on two effects, which are measured in relation to an increasing sarcomere length (L) at a region of decreasing cross-bridges overlap: The first effect is the linear decrease in isometric force (Fo), when neglecting the creep-phase. The second effect is the constant value of unloaded shortening velocity (Vo) [2]. The AS hypothesis anticipates both effects by taking the hydraulic tension ( $\mathrm{Po}$ ) and the sarcomere volume $\left(\mathrm{N}=\mathrm{L}^{*} \mathrm{~A}\right)$ to be independent of $\mathrm{L}$, and by reasonably assuming an inverse relation between the flow-rate $\left(\mathrm{FR}=\mathrm{Vo}^{*} \mathrm{~A}\right)$ and $\mathrm{L}$ at a decreasing cross-bridges overlap. Then:

$$
\begin{gathered}
\mathrm{Fo}=\mathrm{Po} * \mathrm{~A}=\mathrm{Po} * \mathrm{~N} / \mathrm{L}=\text { constant } * 1 / \mathrm{L} \\
\mathrm{Vo}=\mathrm{FR} / \mathrm{A}=\mathrm{FR} * \mathrm{~L} / \mathrm{N}=\text { constant }
\end{gathered}
$$

Notice that at a constant overlap, FR is constant, so that Vo is predicted to increase with L, as observed.

6.3.14. The sub-pressure development of $1 \mathrm{kgwt} / \mathrm{cm}^{2}$ in about half a sarcomere volume also explains the observed initial effect of bulk expansion according to the coefficient of water compression (Supplementary Box 2).

6.3.15. The opposite circulatory propulsion of AS along actin and myosin filaments keeps them well extended for optimal sliding.

6.3.16. Mechanical energy absorption and unbalanced heat release are related, respectively, to stretching and relaxation of serial and transverse elastic elements (Supplementary Box 2). Thus, during isotonic shortening, the heat released per unit length due to ongoing relaxation of transverse elastic elements depends on the load. This heat is quantitatively related to the so-called "heat of shortening" [86].

6.3.17. Another unbalanced energy component is quantitatively related to entropy changes of water associated with either sub-pressure development or relaxation within each sarcomere throughout an isometric cycle of hydraulic compression (Supplementary Box 2). This bulk baro-entropic reversible heat production cancels out during closed cycles, and it accounts to the "thermo-elastic heat" [87]. The baro-entropic component is quantitatively related to the coefficient of thermal water expansion. It is therefore predicted to change sign above and below $4^{\circ} \mathrm{C}$, as observed (Supplementary Box 2).

These unbalanced heat components, and the tension creep phase mentioned above, are not taken into account in the following treatment.

\section{Quantitative formulation of striated muscle contraction (Suppl. Box 3, Suppl. Workbook 1)}

Five energetic parameters of striated muscle contraction are directly related to the quantitative aspects of the pwason model in the pure water phase (Supplementary Box 1):

1. The electro-mechano-chemical power of ATP hydrolysis is related to the free energy of $\mathrm{H}_{2} \mathrm{O}-\mathrm{H}^{+}$, namely: $\Delta \mathrm{E}=0.5$ proton*volt per ATP molecule $=11.5 \mathrm{kcal}$ per mole ATP (See Eq.3).

2. The general isometric tension, $P o=1 \mathrm{kgwt} / \mathrm{cm}^{2}$, is related to the kinetic energy density of water dimer precession. The same isometric tension is produced also by smooth muscle [88]. 
3. The maximal possible value of unloaded shortening velocity per unit muscle length is V1o (max) $=25$ unit length $/ \mathrm{sec}$.

4. The maximal possible value of power density in unloaded or isometric contraction is Hh1o ( $\max$ ) $=2.5 \mathrm{Watt} / \mathrm{cm}^{3}$.

5. The friction coefficient per unit volume is given by the following expression: $\eta_{1}=$ Po/V1o $(\max )=40$ grwt $\cdot \mathrm{cm} \cdot \mathrm{sec} / \mathrm{cm}^{3}=4 \mathrm{mJoule} \cdot \mathrm{sec} / \mathrm{cm}^{3}$.

The process of hydraulic compression of a single sarcomere is further formulated in Supplementary Box 3. The normalized power-balance equation describes the mechanical (Hm) plus heat $(\mathrm{Hq})$ components, and the input hydrolytic (Hh) component (Eq.6i), where $\mathrm{Hq}$ is composed of translation and circulation contributions of AS. The corresponding normalization factors are: the isometric tension, $\mathrm{Po}=1 \mathrm{kgwt} / \mathrm{cm}^{2}$; the unloaded shortening velocity per unit length, $\mathrm{V} 1 \mathrm{o}$; and the isometric power density for $\mathrm{Hm}=0$, Hh1o. By the following argument we prove that Hh1o has the same value in isometric and unloaded contractions. The soliton's life-time of $20 \mathrm{msec}$ may be reduced upon external work production, thus increasing the hydrolytic rate, as observed in the Fenn-Effect. This effect is introduced in its differential and integral forms in Eq.6ii. A general expression is thereby obtained for the normalized force-velocity (P-V) relation in Eq.6iii. The single parameter, $\boldsymbol{a}$, has several meaningful expressions in Eq. 6iv, and its value is proportional to the P-V curvature:

$$
\begin{gathered}
\mathrm{Hm}+\mathrm{Hq}=\mathrm{Hh} \\
\mathrm{a} * \mathrm{P} * \mathrm{~V}+\mathrm{V}^{2}+\mathrm{P}^{2}=\mathrm{Hh} \\
\mathrm{dHh} / \mathrm{dHm}=-\mathrm{dHq} / \mathrm{dHm} \rightarrow \mathrm{Hh}=1+\mathrm{Hm} / 2 ; \mathrm{Hq}=1-\mathrm{Hm} / 2 \\
\mathrm{a}^{*} \mathrm{P} * \mathrm{~V}+\mathrm{V}^{2}+\mathrm{P}^{2}=1+0.5 \mathrm{a} * \mathrm{P} * \mathrm{~V} \\
\mathrm{a}=\mathrm{Po} * \mathrm{~V} 1 \mathrm{o} / \mathrm{Hc} 1 \mathrm{o}=\mathrm{Po} /(\eta 1 * \mathrm{~V} 1 \mathrm{o})=\mathrm{V} 1 \mathrm{o}(\max ) / \mathrm{V} 1 \mathrm{o}=25 / \mathrm{V} 1 \mathrm{o}=(\mathrm{Hh} 1 \mathrm{o}(\max ) / \mathrm{Hh} 1 \mathrm{o}){ }^{1 / 2}
\end{gathered}
$$

where Hh1o $=\eta 1 * \mathrm{~V} 1 \mathrm{o}^{2}=4 * \mathrm{~V} 1 \mathrm{o}^{2} \mathrm{mWatt} / \mathrm{cm}^{3}$.

Analytical solution of Eq.6iii, for various values of $\boldsymbol{a}$, yields the whole spectrum of power balance and force-velocity relations for isotonic contraction (Fig.3a, b). Kinetic profiles of isometric contraction against elastic elements of relative compliance $\boldsymbol{C}$ are obtained by numerical integration of the P-V relation (Fig.3c). (Thus, an effective internal compliance and power input of a given muscle can be evaluated.) The variables of closed cycles of twitch contraction are displayed against the relative tension attained by various elastic loads during the twitch. The twitch duration is related to an effective calcium regulation due to a whole-sarcomere-volume circulation of AS. The optimum total energy values of maximum Eh and Em, and minimum Eq, are obtained at about 0.5Po. Equal but oppositely directed changes are obtained for Eh and Eq with respect to Eho. The Eho value is found to be equal for the unloaded twitch and for the fully developed isometric twitch (Fig.3d). This theoretical profile is closely verified by measurements of isotonic twitch contractions [89]. The high efficiency values are further verified when taking into account the surplus of mechanical energy consumed and the heat released by internal elastic elements (longitudinal as well as transversal), while the baro-entropic component is canceled throughout the closed cycle. A simple analysis of Eq.6iii, compared to the force-velocity relation of A.V. Hill $[87,90]$ is included in the Supplementary Interactive Workbook, 
where all the computational procedures are demonstrated. The present model may become a new useful tool for experts in muscle contraction.
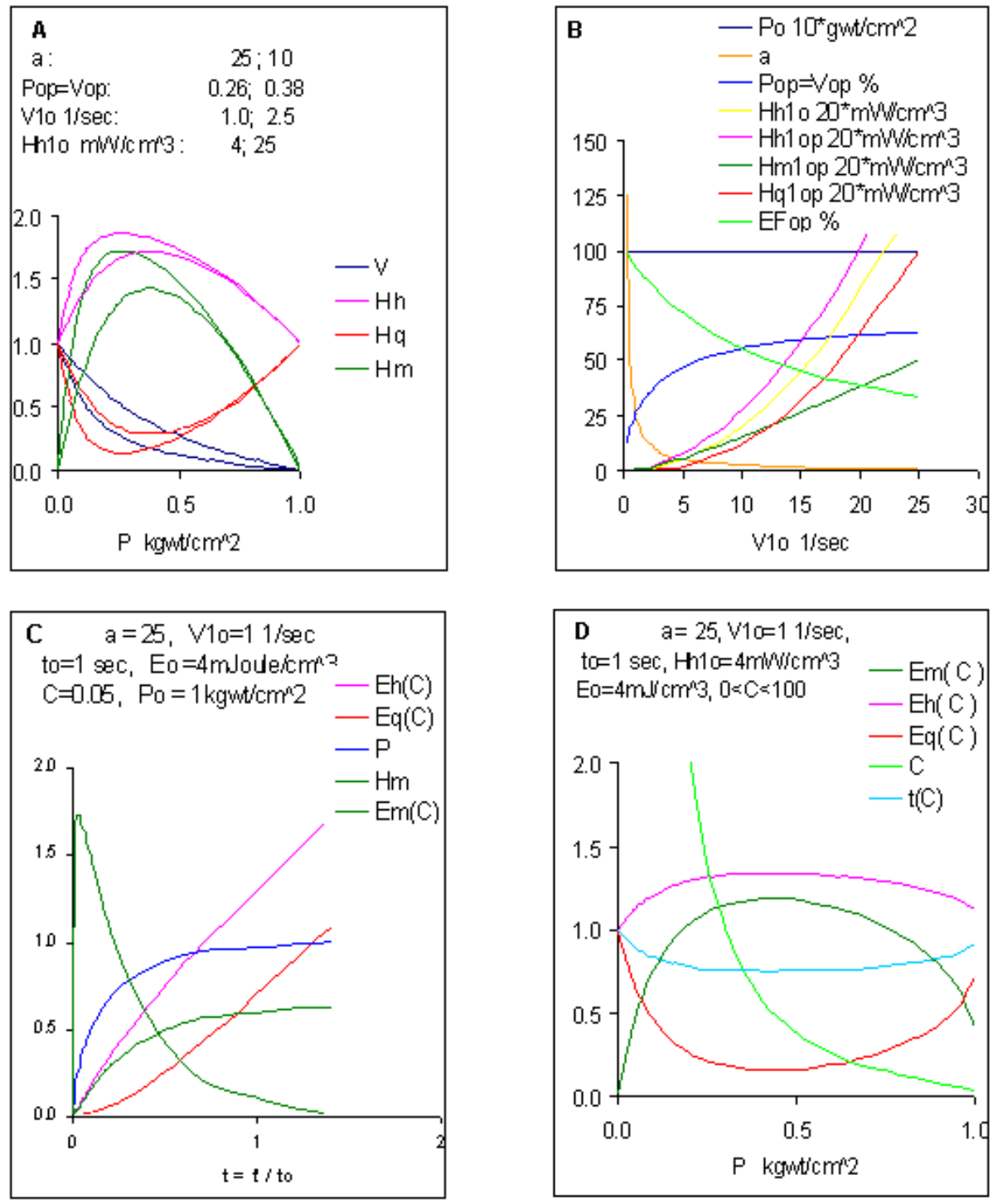

Figure 3. Force-Velocity and Power-Balance relations in isotonic and isometric contractions (see also Supplementary Box 3).

(a) Isotonic Force-Velocity and Power-Balance relations for two values of $\boldsymbol{a}$.

(b) Quantitative spectrum of the isotonic variables versus V1o.

(c) Kinetic profiles of isometric contraction against a load of compliance C.

(d) Spectrum of compliance-dependent variables in isometric twitch contractions, versus the final tension.

\section{Summary}

Electrical, chemical, and hydraulic aspects of the AS model were consistently examined, covering a wide spectrum of novel and testable implications to the physics, chemistry and biology of water. The 
detailed presentation calls for critical experimental and theoretical investigations, as well as for engineering applications.

\section{Acknowledgment}

I would like to thank Ilia Stambler for manuscript editing, and Avshalom Tirosh for the videoanimations. A special appreciation is raised for the critical inspiration of the reviewers.

\section{References}

1. Szent-Gyorgyi, A.G. The early history of the biochemistry of muscle contraction. J. Gen. Physiol. 2004, 123, 631-641.

2. Huxley, A.F. Mechanics and models of the myosin motor. Philos. Trans. R. Soc. Lond. B Biol Sci. 2000, 355, 433-440.

3. Huxley, H.E. Molecular basis of contraction in cross-striated muscles. In The Structure and Function of Muscle; Bourne, G.H., Ed.; Academic Press: New York, 1973; Vol.1, pp.301-387.

4. Tirosh, R.; Oplatka, A.; Chet, I. Motility in a "cell sap" of the slime mold physarum polycephalum. FEBS Lett. 1973, 34, 40-42.

5. Oplatka, A.; Tirosh, R. Active streaming in actomyosin solutions. Biochim. Biophys. Acta. 1973, 305, 684-688.

6. Cohen, I.; Tirosh, R.; Oplatka, A. Active streaming in human thrombosthenin solutions. Pflugers Arch. 1974, 352, 81-85.

7. Oplatka, A.; Borejdo, J.; Gadasi, H.; Tirosh, R.; Liron, N.; Reisler, E. Contraction of Glycerinated Muscle Fibers, Myofibrils and Actin Threads Induced by Water-Soluble Myosin Fragments. Proc. 9th FEBS Meeting. 1974, pp. 41-46.

8. Oplatka, A.; Gadasi, H.; Tirosh, R.; Lamed, Y.; Muhlrad, A.; Liron, N. Demonstration of mechanochemical coupling in systems containing actin, ATP and non-aggregating active myosin derivatives. J. Mechanochem. Cell Motil. 1974, 2, 295-306.

9. Oplatka, A.; Borejdo, J.; Gadasi, H. Tension development in stretched glycerinated muscle fibers and contraction of 'ghost' myofibrils induced by irrigation with heavy meromyosin. FEBS Lett. 1974, 45, 55-58.

10. Oplatka, A.; Gadasi, H.; Borejdo, J. The contraction of "ghost" myofibrils and glycerinated muscle fibers irrigated with heavy meromyosin subfragment-1. Biochem. Biophys. Res. Commun. 1974, 58, 905-912.

11. Borejdo, J.; Oplatka, A. Tension development in skinned glycerinated rabbit psoas fiber segments irrigated with soluble myosin fragments. Biochim. Biophys. Acta. 1976, 440, 241-258.

12. Tirosh, R.; Oplatka, A. Active streaming against gravity in glass microcapillaries of solutions containing acto-heavy meromyosin and native tropomyosin. J. Biochem. 1982, 91, 1435-1440.

13. Tirosh, R.; Low, W.Z.; Oplatka, A. Translational motion of actin filaments in the presence of heavy meromyosin and MgATP as measured by Doppler broadening of laser light scattering. Biochim. Biophys. Acta. 1990, 1037, 274-280.

14. Tirosh, R. Ph.D. Thesis: Elementary Aspects of the Mechano-Chemical Coupling in the Actomyosin System. The Weizmann Institute of Science: Rehovot, 1978 (in Hebrew). 
15. Tirosh, R.; Liron, N.; Oplatka, A. A hydrodynamic mechanism for muscular contraction. In CrossBridge Mechanism in Muscle Contraction, Proceedings of the International Symposium on the Current Problems of Sliding Filament Model and Muscle Mechanics, Tokyo, Japan, 1978. University of Tokyo Press: Tokyo, 1979; pp. 593-609.

16. Cairns, R.B.; Harrison, H.; Schoen, R.I. Dissociative Photoionization of $\mathrm{H}_{2}$ O. J. Chem. Phys. 1971, 55, 4886-4889.

17. Head-Gordon, T.; Hura, G. Water structure from scattering experiments and simulation. Chem Rev. 2002, 102, 2651-2670.

18. Bakker, H.J.; Nienhuys, H.K. Delocalization of protons in liquid water. Science 2002, 297, 587590.

19. Kashtanov, S.; Augustsson, A.; Luo, Y.; Guo, J.H.; Sathe, C.; Rubensson, J.E.; Siegbahn, H.; Nordgren, J; Agren, H. Local structures of liquid water studied by x-ray emission spectroscopy. Phys. Rev. B. 2004, 69, 024201.

20. Wernet, Ph.; Nordlund, D.; Bergmann, U.; Cavalleri, M.; Odelius, M.; Ogasawara, H.; Naslund, L.A.; Hirsch, T.K.; Ojamae, L.; Glatzel, P.; Pettersson, L.G.M.; Nilsson, A. The Structure of the First Coordination Shell in Liquid Water. Science 2004, 304, 995-999.

21. Bushuev, Y.G.; Davletbaeva, S.V.; Muguet, F. The 3-Attractor Water Model: Monte-Carlo simulations with a new, effective 2-Body potential (BMW). Molecules 2003, 8, 226-242.

22. Wilkie, D.R;. Heat work and phosphorylcreatine break-down in muscle. J. Physiol. 1968, 195, 157183.

23. Mohammed OF, Pines D, Dreyer J, Pines E, Nibbering ET; Sequential proton transfer through water bridges in acid-base reactions. Science 2005, 310, 83-86.

24. Kaufmann, K.; Silman, I. The induction by protons of ion channels through lipid bilayer membranes. Biophys. Chem. 1983, 18, 89-99.

25. Soper, A.K. Thermodynamics. Water and ice. Science 2002, 297, 1288-1289.

26. Giovambattista, N.; Stanley, H.E.; Sciortino, F. Relation between the high density phase and the very-high density phase of amorphous solid water. Phys. Rev. Lett. 2005, 94, 107803.

27. Drost-Hansen, W. Temperature effects on cell-functioning--a critical role for vicinal water. Cell Mol. Biol. (Noisy-le-grand). 2001, 47, 865-883

28. "Waves of the Sea." Encyclopedia Britannica 1970, 23, 316-317.

29. Allen, J E. The Early History of Solitons (Solitary Waves). Phys. Scr. 1998, 57, 436-441.

30. Olson, E.S. Direct measurement of intra-cochlear pressure waves. Nature 1999, 402, 526-529.

31. La Porta, A.; Voth, G.A.; Crowford, A.M.; Alexander, J.; Bodenschatz, E. Fluid particles accelerations in fully developed turbulence. Nature 2001, 409, 1017-1019.

32. Flannigan, D.J.; Suslick, K.S. Plasma formation and temperature measurement during singlebubble cavitation. Nature 2005, 434, 52-55.

33. Holmes, K.C.; Schroder, R.R.; Sweeney, H.L.; Houdusse, A. The structure of the rigor complex and its implications for the power stroke. Philos. Trans. R. Soc. Lond. B. Biol Sci. 2004, 359, 18191828.

34. Lymn, R.W.; Taylor, E.W. Mechanism of adenosine triphosphate hydrolysis by actomyosin. Biochemistry 1971, 10, 4617-4624. 
35. Sleep, J.A.; Hackney, D.D.; Boyer, P.D. The equivalence of phosphate oxygens for exchange and the hydrolysis characteristics revealed by the distribution of $\left[{ }^{18} \mathrm{O}\right] \mathrm{Pi}$ species formed by myosin and actomyosin ATPase. J. Biol. Chem. 1980, 255, 4094-4099.

36. White, H.D.; Belknap, B.; Webb, M.R. Kinetics of nucleoside triphosphate cleavage and phosphate release steps by associated rabbit skeletal actomyosin, measured using a novel fluorescent probe for phosphate. Biochemistry 1997, 36, 11828-11836.

37. Takagi, Y.; Shuman, H.; Goldman, Y.E. Coupling between phosphate release and force generation in muscle actomyosin. Philos. Trans. R. Soc. Lond. B. Biol. Sci. 2004, 359, 1913-1920.

38. Wells, A.L.; Lin, A.W.; Chen, L.Q.; Safer, D.; Cain, S.M.; Hasson, T.; Carragher, B.O.; Milligan, R.A.; Sweeney, H.L. Myosin VI is an actin-based motor that moves backwards. Nature 1999, 401, 505-508.

39. De La Cruz, E.M.; Ostap, E.M. Relating biochemistry and function in the myosin superfamily. Curr. Opin. Cell Biol. 2004, 16, 61-67.

40. Forkey, J.N.; Quinlan, M.E.; Shaw, M.A.; Corrie, J.E.; Goldman, Y.E. Three-dimensional structural dynamics of myosin $\mathrm{V}$ by single-molecule fluorescence polarization. Nature 2003, 422, 399-404.

41. Fletcher, D.A.; Theriot, J.A. An introduction to cell motility for the physical scientist. Phys. Biol. 2004, 1, T1-10.

42. Mitchell, P. A commentary on alternative hypotheses of protonic coupling in the membrane systems catalysing oxidative and photosynthetic phosphorylation. FEBS Lett. 1977, 78, 1-20.

43. Harold, F.M. Gleanings of a chemiosmotic eye. BioEssays 2001, 23, 848-855.

44. Kaim, G.; Dimroth, P. ATP synthesis by F-type ATP synthase is obligatorily dependent on the transmembrane voltage. EMBO J. 1999, 18, 4118-4127.

45. Boyer, P.D. A research journey with ATP synthase. J. Biol. Chem. 2002, 277, 39045-39061.

46. Noji, H., Yasuda, R., Yoshida, M. \& Kinosita, K. Jr Direct observation of the rotation of F1ATPase. Nature 1997, 386, 299-302.

47. Rondelez, Y.; Tresset, G.; Nakashima, T.; Kato-Yamada, Y.; Fujita, H.; Takeuchi, S.; Noji, H. Highly coupled ATP synthesis by F1-ATPase single molecules. Nature 2005, 433, 773-777.

48. Berg, H.C. The rotary motor of bacterial flagella. Annu. Rev. Biochem. 2003, 72, 19-54,

49. Smith, C.A.; Rayment, I. Active site comparisons highlight structural similarities between myosin and other P-loop proteins. Biophys. J. 1996, 70, 1590-602.

50. Tucker, W.C.; Schwarz, A.; Levine, T.; Du, Z.; Gromet-Elhanan, Z.; Richter, M.L.; Haran, G. Observation of Calcium-dependent unidirectional rotational motion in recombinant photosynthetic F1-ATPase molecules. J. Biol. Chem. 2004, 279, 47415-47418.

51. Haumann, M.: Liebisch, P.; Muller, C.; Barra, M.; Grabolle, M.; Dau, H. Photosynthetic $\mathrm{O}_{2}$ formation tracked by time-resolved x-ray experiments. Science 2005, 310, 1019-1021.

52. Kitamura, K.; Tokunaga, M.; Iwane, A.H.; Yanagida, T. A single myosin head moves along an actin filament with regular steps of 5.3 nanometres. Nature 1999, 397, $129-134$.

53. Sakamoto, T.; Amitani, I.; Yokota, E.; Ando, T. Direct observation of processive movement by individual myosin V molecules. Biochem. Biophys. Res. Commun. 2000, 272, 586-590. 
54. Ali, M.Y.; Homma, K.; Iwane, A.H.; Adachi, K.; Itoh, H.; Kinosita, K.Jr.; Yanagida, T.; Ikebe, M. Unconstrained steps of myosin VI appear longest among known molecular motors. Biophys. J. 2004, 86, 3804-3810.

55. Rock, R.S.; Ramamurthy, B.; Dunn, A.R.; Beccafico, S.; Rami, B.R.; Morris, C.; Spink, B.J.; Franzini-Armstrong, C.; Spudich, J.A.; Sweeney, H.L. A flexible domain is essential for the large step size and processivity of myosin VI. Mol. Cell. 2005, 17, 603-609.

56. Riveline, D.; Ott, A.; Julicher, F.; Winkelmann, D.A.; Cardoso, O.; Lacapere, J.J.; Magnusdottir, S.; Viovy, J.L.; Gorre-Talini, L.; Prost, J. Acting on actin: the electric motility assay. Eur. Biophys. J. 1998, 27, 403-408.

57. Landesberg, A.; Landesberg, Y.; Sideman, S.; Ter Keurs, H.E. Molecular motion and cardiac muscle motor dynamics. Ann. NY Acad. Sci. 2002, 972, 119-126.

58. Kamiya, N.; Abe, S. Bioelectric phenomena in the myxomycete plasmodium and their relation to protoplasmic flow. J. Colloid. Sci. 1950, 5, 149.

59. Tirosh, R. Axoplasmic transport and the nerve impulse explained by cytoplasmic axial flux of energetic protons along actin filaments. In Biological Structures and Coupled Flows, Proceedings of the Symposium Dedicated to Aharon Katzir Katchalsky; Oplatka A., Balaban, M., Eds.; Intern. Sci. Serv: Rehovot, 1983; pp. 397-400.

60. Tasaki, I.; Iwasa, K. Further studies of rapid mechanical changes in squid giant axon associated with action potential production. Jpn. J. Physiol. 1982, 32, 505-518.

61. Tasaki, I. Rapid structural changes in nerve fibers and cells associated with their excitation processes. Jpn. J. Physiol. 1999, 49, 125-138.

62. Mermall, V.; Post, P.L.; Mooseker, M.S. Unconventional myosins in cell movement, membrane traffic, and signal transduction. Science 1998, 279, 527-533.

63. Zakon, H.H.; Unguez, G.A. Development and regeneration of the electric organ. J. Exp. Biol. 1999, 202, 1427-1434.

64. Nuccitelli, R.; Poo, M.M.; Jaffe, L.F. Relations between ameboid movement and membranecontrolled electrical currents. J. Gen. Physiol. 1977, 69, 743-763.

65. Charras, G.T.; Yarrow, J.C.; Horton, M.A.; Mahadevan, L.; Mitchison, T.J. Non-equilibration of hydrostatic pressure in blebbing cells. Nature 2005, 435, 365-369.

66. Chapman-Andresen, C. Endocytosis in freshwater amebas. Physiol. Rev. 1977, 57, 371-385.

67. Tirosh, R.; Berke, G. Immune cytolysis viewed as a stimulatory process of the target. Adv. Exp. Med. Biol. 1985, 184, 473-492.

68. Giardini, P.A.; Fletcher, D.A.; Theriot, J.A. Compression forces generated by actin comet tails on lipid vesicles. Proc. Natl. Acad. Sci. USA. 2003, 100, 6493-6498.

69. Tirosh, R.; Degani, H.; Berke, G. Prelytic reduction of high-energy phosphates induced by antibody and complement in nucleated cells. ${ }^{31} \mathrm{P}-\mathrm{NMR}$ study. Complement 1984, 1, 207-212

70. Leduc, C.; Campas, O.; Zeldovich, K.B.; Roux, A.; Jolimaitre, P.; Bourel-Bonnet, L.; Goud, B.; Joanny, J.F.; Bassereau, P.; Prost, J. Cooperative extraction of membrane nanotubes by molecular motors. Proc. Natl. Acad. Sci. USA. 2004, 101, 17096-17101.

71. Tuvia, S.; Levin, S.; Bitler, A.; Korenstein, R. Mechanical fluctuations of the membrane-skeleton are dependent on F-Actin ATPase in human erythrocytes. J. Cell Biol. 1998, 141, 1551-1561. 
72. Girard, P.; Prost, J.; Bassereau, P. Passive or active fluctuations in membranes containing proteins. Phys. Rev. Lett. 2005, 94, 088102.

73. Bernheim-Groswasser, A.; Prost, J.; Sykes, C. Mechanism of actin-based motility: a dynamic state diagram. Biophys. J. 2005, 89, 1411-1419.

74. Uyeda, T.Q.; Nagasaki, A. Variations on a theme: the many modes of cytokinesis. Curr. Opin. Cell. Biol. 2004, 16, 55-60.

75. Huxley, H.E. A personal view of muscle and motility mechanisms. Annu. Rev. Physiol. 1996, 58, 1-19.

76. Franzini-Armstrong, C. Membraneous systems in muscle fibers. In The Structure and Function of Muscle; Bourne, G.H., Ed.; Academic Press: New York, 1973; Vol. 2, pp. 531-619.

77. Piazzesi, G.; Reconditi, M.; Linari, M.; Lucii, L.; Sun, Y.B.; Narayanan, T.; Boesecke, P.; Lombardi, V.; Irving, M. Mechanism of force generation by myosin heads in skeletal muscle. Nature 2002, 415, 659-661.

78. Tirosh, R. $1 \mathrm{kgf} / \mathrm{cm} 2$ - The isometric tension of muscle contraction: implications to cross-bridge and hydraulic mechanisms. Adv. Exp. Med. Biol. 1984, 170, 531-539.

79. Edman, K.A.P. The force bearing capacity of frog muscle fibres during stretch: its relation to sarcomere length and fibre width. J. Physiol. 1999, 519, 515-526.

80. ter Keurs, H.E.; Iwazumi, T.; Pollack, G.H. The length-tension relation in skeletal muscle. J. Gen. Physiol. 1978, 4, 565-592.

81. Rassier, D.E.; Herzog, W.; Pollack, G.H. Dynamics of individual sarcomeres during and after stretch in activated single myofibrils. Proc. R. Soc. Lond. B. Biol. Sci. 2003, 270, 1735-1740.

82. Katz, B. The relation between force and speed in muscular contraction, J. Physiol. 1939, 96, 45-64.

83. Goldman, Y.E.; Hibberd, M.G.; McCray, J.A.; Trentham, D.R. Relaxation of muscle fibres by photolysis of caged ATP. Nature 1982, 300, 701-705.

84. Delay, M.J.; Ishide, N.; Jacobson, R.C.; Pollack, G.H.; Tirosh, R. Stepwise shortening: analysis by high-speed cinemicrography. Science 1981, 213,1523-1525

85. Dickinson, M.; Farman, G.; Frye, M.; Bekyarova, T.; Gore, D.; Maughan, D.; Irving, T. Molecular dynamics of cyclically contracting insect flight muscle in vivo. Nature 2005, 433, 330-334.

86. Hill, A.V. The effect of load on the heat of shortening of muscle. Proc. R. Soc. Lond.B. Biol. Sci. 1964, 159, 297-318.

87. Woledge, R.C.; Curtin, N.A.; Homsher, E. Energetic Aspects of Muscle Contraction. Monogr. Physiol. Soc. 1985, 41, 1-357.

88. Csapo, A.I. Smooth muscle. In The Structure and Function of Muscle; Bourne, G.H., Ed.; Academic Press: New York, 1973; Vol.2, p. 16.

89. Hill, AV. The variation of total heat production in a twitch with velocity of shortening. Proc. $R$. Soc. Lond. B. Biol. Sci. 1964, 159, 596-605.

90. Hill, A.V. First and Last Experiments in Muscle Mechanics. Cambridge University Press: London, 1970. 


\section{Supplementary Materials}

Box 1. Protonic induction and hydraulic action of a water soliton (Fig.1 A,B; Movies 1,2)

Box 2. Heat contributions due to elastic and baro-entropic components in a half sarcomere

Box 3. Mechano-chemical conversion into hydraulic compression by active streaming in isotonic and isometric contractions (Fig.3; Interactive Workbook 1)

Table 1. Molecular distances in a pair of dimers (Fig.1a)

Movie 1. Proton-induced water soliton. A ballistic $\mathrm{H}^{+}$is released from $\mathrm{H}_{2} \mathrm{O}-\mathrm{H}^{+}$with a kinetic energy of 0.5 proton*volt, which corresponds to an initial velocity of $10 \mathrm{~km} / \mathrm{sec}$. By coherent exchange of microwave photons during $10^{-10} \mathrm{sec}$, along a straight path of $500 \mathrm{~nm}$, it induces cooperative precession of 13300 electrically polarized water-molecule dimers. The dimers reorganize into non-radiating octal rings that compose a persistent rowing water soliton.

Movie 2. Rowing soliton. By peripheral rowing-like action, the water soliton continues to propagate during $20 \mathrm{msec}$ at a velocity of $25 \mu \mathrm{m} / \mathrm{sec}$, and is able to generate and overcome a maximal pressurehead of $1 \mathrm{kgwt} / \mathrm{cm}^{2}$.

Workbook 1. An interactive workbook for quantitative extraction of muscle contraction variables. Values in color-highlighted cells can be modified (see notes in the workbook).

A. P-V-H relations in isotonic tetanus (Compare to Hill's Equation).

B. Spectrum of P-V-H isotonic parameters.

C. Development of isometric tetanus against an elastic load of compliance C.

Also shown: The theoretical equation for isotonic contraction, the Fenn Effect relation, and the relation between Hill's "heat of maintenance" and the theoretical isometric heat.

All supplementary material is available from http://www.mdpi.org/ijms/papers/i7090320/

(C) 2006 by MDPI (http://www.mdpi.org). Reproduction is permitted for noncommercial purposes. 